\title{
Variability in the timeliness of interventional radiology availability for angioembolization of hemodynamically unstable pelvic fractures: a prospective survey among U.S. level I trauma centers
}

Stephanie Jarvis ${ }^{1}$, Alessandro Orlando ${ }^{1}$, Benoit Blondeau ${ }^{2,3}$, Kaysie Banton ${ }^{4}$, Cassandra Reynolds ${ }^{4}$, Gina M. Berg ${ }^{5}$, Nimesh Patel ${ }^{6}$, Michael Kelly ${ }^{7}$, Matthew Carrick ${ }^{8}$ and David Bar-Or ${ }^{1,4^{*}}$

\begin{abstract}
Background: Patients with hemodynamically unstable pelvic fractures have high mortality due to delayed hemorrhage control. We hypothesized that the availability of interventional radiology (IR) for angioembolization may vary in spite of the mandated coverage at US Level I trauma centers, and that the priority treatment sequence would depend on IR availability.

Methods: This survey was designed to investigate IR availability and pelvic fracture management practices. Six email invitations were sent to 158 trauma medical directors at Level I trauma centers. Participants were allowed to skip questions and irrelevant questions were skipped; therefore, not all questions were answered by all participants. The primary outcome was the priority treatment sequence for hemodynamically unstable pelvic fractures. Predictor variables were arrival times for IR when working off-site and intervention preparation times. Kruskal-Wallis and ordinal logistic regression were used; alpha $=0.05$.

Results: Forty of the 158 trauma medical directors responded to the survey (response rate: $25.3 \%$ ). Roughly half of participants had 24-h on-site IR coverage, 24\% (4/17) of participants reported an arrival time $\geq 31$ min when IR was on-call. 46\% (17/37) of participants reported an IR procedure setup time of 31-120 min. Arrival time when IR was working off-site, and intervention preparation time did not significantly affect the sequence priority of angioembolization for hemodynamically unstable pelvic fractures.
\end{abstract}

Conclusions: Trauma medical directors should review literature and guidelines on time to angioembolization, their arrival times for IR, and their procedural setup times for angioembolization to ensure utilization of angioembolization in an optimal sequence for patient survival.

Keywords: Pelvic fracture management, Interventional radiology, Angioembolization, Resuscitative endovascular balloon occlusion of the aorta

\footnotetext{
* Correspondence: davidbme49@gmail.com

${ }^{1}$ Trauma Research, LLC, 383 Corona St. \#319, Denver, CO 80218, USA

${ }^{4}$ Swedish Medical Center, 501 E Hampden Ave, Englewood, CO 80113, USA

Full list of author information is available at the end of the article
}

(C) The Author(s). 2019 Open Access This article is distributed under the terms of the Creative Commons Attribution 4.0 International License (http://creativecommons.org/licenses/by/4.0/), which permits unrestricted use, distribution, and reproduction in any medium, provided you give appropriate credit to the original author(s) and the source, provide a link to the Creative Commons license, and indicate if changes were made. The Creative Commons Public Domain Dedication waiver (http://creativecommons.org/publicdomain/zero/1.0/) applies to the data made available in this article, unless otherwise stated. 


\section{Background}

Pelvic fracture management is one of the most complex treatment strategies [1]. Published guidelines offer varying approaches to care for hemodynamically unstable pelvic fractures [2-6]. The World Society of Emergency Surgeons (WSES) and Western Trauma Association (WTA) recommend selective angioembolization after pelvic packing [2, 3]. Eastern Association for the Surgery of Trauma (EAST) and Advanced Trauma Life Support (ATLS) suggest angioembolization after circumferential compression device application [5, 6]. Trauma Quality Improvement Program (TQIP) [4] utilizes angioembolization after external fixation and pelvic packing, or last when in extremis. There remains a high level of ambiguity on the optimal management of patients with hemodynamic unstable pelvic fractures across guidelines [2-6].

It is known that the time from presentation to angiography affects mortality in cases where angioembolization is needed [7]. Tanizaki et al. found a 4-fold increase in mortality rates for patients who went to angiography $60 \mathrm{~min}$ after arrival when compared to those who went within $60 \mathrm{~min}$ [7]. This is at least part of the reason that the American College of Surgeons (ACS) requires an interventional radiologist available within $30 \mathrm{~min}$ at Level I trauma centers [8]. Although, it has been reported that not all Level I trauma centers have IR on-site, the full extent of IR availability has not been described; therefore it is unclear if angiography within $1 \mathrm{~h}$ of arrival is possible [9].

\section{Methods}

This anonymous cross-sectional survey of 158 trauma medical directors at United States ACS-verified Level I trauma centers was approved by the Western Institutional Review Board. The contact list was derived from the ACS website, individual trauma center's websites, and via telephone. To view the invitation list, view the Appendix. Coauthors piloted the web-based survey prior to its online dissemination through SurveyMonkey Inc. (San Mateo, California; www.surveymonkey.com). Six invitations, that contained the approved partial waiver of consent, were emailed from March 1, 2018 to June 26, 2018. Participants were called to verify email receipt if they had not responded upon sending the final two invitations. No compensation was provided, and participation was voluntary. Trauma medical directors or an assigned colleague completed the survey and are referred to as "participants".

The study hypotheses were 1) that IR was not on-site and prepared for intervention within $60 \mathrm{~min}$, and 2) arrival times for IR when working off-site and the time for IR to prepare for intervention would be associated with the priority treatment sequence for angioembolization. The survey included 46 questions regarding IR availability and pelvic fracture management practices. To view questions pertaining to this paper, visit: http://bit.ly/SurveyIR. Irrelevant questions were skipped based on prior responses using SurveyMonkey's 'skip logic', and participants could skip any question; therefore, there are missing responses for individual questions. Analysis was completed on SAS 9.4 (Cary, NC) software. Categorical data were summarized as counts and proportions. The median (interquartile range [IQR]) sequence for angioembolization was compared by both the arrival time for IR, and by the time for IR to prepare for intervention using the Kruskal-Wallis test. Ordinal logistic regression was used to determine if the arrival time for IR, or the time for IR to prepare for intervention was associated with the priority treatment sequence for angioembolization. All hypothesis tests were two-tailed with an alpha of 0.05 .

\section{Results}

The response rate was $25 \%$ (40/158). Of the survey responses, 90\% (36/40) completed and 10\% (4/40) partially completed the survey; all responses were included. Participating Level I trauma centers' characteristics have been reported [10]. The median (IQR) survey completion time was $11 \mathrm{~min}(8,21)$. No pelvic fracture protocol was implemented at $28 \%(11 / 40)$ of participating Level I trauma centers (Table 1). The most common pelvic fracture guideline followed was the EAST guideline (23\% [11/40]). A majority of participants preferred using angioembolization before pelvic packing (63\% [17/27]). Contrast extravasation was the most common angioembolization indicator (60\% [21/35]).

Fifty-four percent (20/37) of the represented Level I trauma centers had 24-h on-site IR coverage (Table 2). The remaining had on-call IR coverage; $13 \%(2 / 16)$ of participants reported IR was on-call for $24 \mathrm{~h} /$ day, and 31\% (5/ 16) reported IR was on-call for $12 \mathrm{~h} /$ day. A majority $(71 \%$ [12/17]) of participants reported a 21-30-min arrival time for IR when on-call. In addition to arrival times, 46\% (17/ 37) of participants reported an IR procedure set-up time of 31-120 min. Most participants provided temporalizing stabilization through circumferential compression devices, pelvic packing, or REBOA while waiting for IR to prepare for intervention (Table 1).

We previously reported the priority treatment sequence for hemodynamically unstable pelvic fractures [10]. The median priority treatment sequence for angioembolization was examined according to the IR arrival time when working off-site and to the time it took IR to prepare for intervention (Table 3). There was no significant relationship between the arrival times, or the intervention preparation time, and median priority sequence of angioembolization. The intervention preparation time, and the arrival time for IR when working off-site, were not significant predictors for the priority treatment sequence of angioembolization, (Table 4). This is evidenced by a lack of significance for these variables as well as a lack of significance in the Hosmer-Lemeshow goodness of fit p-value. 
Table 1 Angiography for Pelvic Fracture Management at Level I Trauma Centers

\begin{tabular}{l} 
Questions and Possible Responses $\%(n) \quad n$ \\
\hline What agency's guideline is your trauma center following for pelvic
\end{tabular}
fracture management?

$\begin{array}{ll}\text { No guideline in place } & 28 \%(11) \\ \text { Eastern Association for the Surgery of Trauma } & 23 \%(9) \\ \text { Hospital developed protocol } & 18 \%(7) \\ \text { Western Trauma Association } & 15 \%(6) \\ \text { Trauma Quality Improvement Program } & 8 \%(3) \\ \text { Advanced Trauma Life Support } & 5 \%(2) \\ \text { Agency not specified } & 5 \%(2)\end{array}$

Does your hospital use both angioembolization and pelvic packing for pelvic fracture management?

Yes

$85 \%(23) \quad 27$

No

$15 \%(4)$

Angioembolization or Pelvic Packing First?

$\begin{array}{ll}\text { Angioembolization } & 63 \%(17) \\ \text { Pelvic packing } & 37 \%(10)\end{array}$

Does your trauma center have a mobile c-arm?

Yes

$100 \%(36) \quad 36$

No

0

Indicators for angioembolization

$\begin{array}{ll}\text { Contrast extravasation } & 60 \%(21) \\ \text { Hemodynamically unstable } & 46 \%(16) \\ \text { Physician's discretion } & 17 \%(6) \\ \text { Hemodynamically stable } & 14 \%(5) \\ \text { APC, LC, or VS fracture pattern } & 9 \%(3) \\ \text { After pelvic packing } & 9 \%(3) \\ \text { After a circumferential compression device } & 9 \%(3) \\ \text { Pelvic hematoma } & 9 \%(3) \\ \text { Requiring ongoing transfusions } & 9 \%(3) \\ \text { After REBOA } & 3 \%(1) \\ \text { Pseudoaneurysm } & 3 \%(1)\end{array}$

When contrast extravasation is absent on computed tomography, but the patient is hemodynamically unstable, is angioembolization considered a treatment option?

$\begin{array}{ll}\text { Yes } & 70 \%(25) \\ \text { No } & 36 \%(11)\end{array}$

What treatment is utilized while waiting for IR to set-up?

$\begin{array}{ll}\text { Circumferential compression device } & 90 \%(35) \\ \text { Pelvic packing } & 64 \%(25) \\ \text { REBOA } & 44 \%(17) \\ \text { Exploratory laparotomy } & 31 \%(12) \\ \text { Other (massive transfusion protocol) } & 3 \%(1)\end{array}$

a Participants allowed to select multiple responses, $I R$ interventional radiology, $R E B O A$ resuscitative endovascular balloon occlusion of the aorta, APC anteriorposterior compression, $L C$ lateral compression, VS vertical shear
Table 2 Interventional Radiology Coverage at Level I Trauma Centers

\begin{tabular}{|c|c|c|}
\hline Questions and Responses & $\%(n)$ & $\mathrm{n}$ \\
\hline \multicolumn{3}{|c|}{$\begin{array}{l}\text { Does the interventional radiology department have on-site coverage } \\
\text { 24-h a day? }\end{array}$} \\
\hline Yes & $54 \%(20)$ & 37 \\
\hline No & $46 \%(17)$ & \\
\hline
\end{tabular}

How many hours per day is there an interventional radiologist available by call only?

$\begin{array}{ll}8 & 13 \%(2) \\ 10 & 19 \%(3) \\ 12 & 31 \%(5) \\ 13 & 6 \%(1) \\ 14 & 13 \%(2) \\ 15 & 6 \%(1) \\ 24 & 13 \%(2)\end{array}$

Approximately how long does it take for an interventional radiologist to arrive when working off-site?

$\begin{array}{ll}0-10 \mathrm{~min} & 0 \\ 11-20 \mathrm{~min} & 6 \%(1) \\ 21-30 \mathrm{~min} & 71 \%(12) \\ \geq 31 \mathrm{~min} & 24 \%(4)\end{array}$

Approximately how long does it take for IR to set-up for angioembolization once an interventional radiologist is on-site?

\begin{tabular}{ll}
$0-30 \mathrm{~min}$ & $54 \%(20)$ \\
$31-60 \mathrm{~min}$ & $35 \%(13)$ \\
$61-120 \mathrm{~min}$ & $11 \%(4)$ \\
$120-180 \mathrm{~min}$ & 0 \\
$>180 \mathrm{~min}$ & 0 \\
\hline
\end{tabular}

$I R$ Interventional radiology

Table 3 Interventional Radiology Arrival and Preparation Times with the Median Treatment Sequence for Angioembolization

\begin{tabular}{|c|c|c|c|}
\hline & Median (IQR) & $n^{a} / N^{b}$ & $p$ \\
\hline \multicolumn{4}{|c|}{ Time for interventional radiologists to arrive } \\
\hline $0^{c}$ & $1(1,3)$ & $8 / 20$ & 0.84 \\
\hline $0-10 \min$ & N/A & $0 / 0$ & \\
\hline $11-20 \min$ & $2(2,2)$ & $0 / 1$ & \\
\hline $21-30 \min$ & $1(1,2)$ & $5 / 12$ & \\
\hline$\geq 31 \mathrm{~min}$ & $1.5(1,2)$ & $1 / 4$ & \\
\hline
\end{tabular}

Time for interventional radiology to prepare for intervention
0-30 min
$1(1,2)$
$8 / 20$
$31-60 \mathrm{~min}$
$1(1,2)$
$5 / 13$
$61-120 \mathrm{~min}$
$2(1,3)$
$1 / 4$

0.72

${ }^{a}$ number of patients who chose to use angioembolization first, ${ }^{b}$ total number of patients responding, ' participants who indicated their interventional radiology department has on-site coverage $24-h$ a day 
Table 4 Odds of Subsequent Priority Sequence of Angioembolization for IR Arrival and Preparation Times

\begin{tabular}{|c|c|c|c|}
\hline & $\mathrm{OR}(\mathrm{Cl})$ & $p$ & $\mathrm{H}-\mathrm{L}$ GOF \\
\hline \multicolumn{4}{|c|}{ Time for interventional radiologists to arrive } \\
\hline $0^{a}$ & Ref. & 0.24 & $<0.0001$ \\
\hline $0-10 \mathrm{~min}$ & N/A & & \\
\hline $11-20 \mathrm{~min}$ & $0.48(0.06,3.92)$ & & \\
\hline $21-30 \mathrm{~min}$ & $0.39(0.15,1.02)$ & & \\
\hline$\geq 31 \mathrm{~min}$ & $1.12(0.27,4.67)$ & & \\
\hline \multicolumn{4}{|c|}{ Time for interventional radiology to prepare for intervention } \\
\hline $0-30 \mathrm{~min}$ & Ref. & 0.06 & $<0.0001$ \\
\hline $31-60 \mathrm{~min}$ & $0.32(0.12,0.84)$ & & \\
\hline $61-120 \mathrm{~min}$ & $0.90(0.29,2.75)$ & & \\
\hline
\end{tabular}

IR interventional radiology, OR odds ratio, $\mathrm{Cl}$ confidence interval, $\mathrm{H}-\mathrm{L}$ GOF Hosmer-Lemmeshow goodness of fit, ${ }^{a}$ participants who indicated their interventional radiology department has on-site coverage $24-h$ a day

\section{Discussion}

This study surveyed 25\% of ACS-verified Level I trauma centers on angiography practices and IR availability to treat hemodynamically unstable pelvic fractures. We failed to reject the null hypotheses; IR availability was variable across Level I trauma centers and did not significantly affect the priority treatment sequence of angioembolization. A majority of participants utilized angioembolization and pelvic packing, supporting the argument that pelvic packing and angioembolization should be complementary, not competitive, as the treatments target either venous or arterial hemorrhages [11]. Angioembolization primarily treats arterial bleeds, representing $10-20 \%$ of hemorrhaging, but cannot treat the majority of hemorrhaging from venous and cancellous sources [2]. Although the priority sequence for angioembolization and pelvic packing continues to be debated, this study observed a reported preference.

The majority of participants used angioembolization before pelvic packing. Contrary to this, it has been suggested that pelvic packing may be more efficient when used before angioembolization as it treats the majority of pelvic hemorrhaging [2]. Predicting the need for angioembolization has proven difficult; applying pelvic packing first allows for identification of the bleed source and determination of the need for angioembolization [3, 9, 11-13]. Additionally, several studies found a shorter time from admission to pelvic packing than angiography [13-16]. The use of angioembolization before pelvic packing may be due to EAST guideline, being the most commonly followed guideline, recommending angioembolization first [5]. Although Cothren et al. [17] stated preperitoneal pelvic packing can supplant angioembolization needs, this study found that most participants utilized angioembolization and prioritized it earlier than other treatment modalities.

It is our observation that a common reason for pelvic packing application is due to excessive wait times for IR.
Despite the prevalence of angioembolization before pelvic packing, roughly half of the responding Level I trauma centers did not have 24-h on-site IR coverage. Furthermore, many participants reported arrival and IR procedure preparation times in excess of $30 \mathrm{~min}$; some as long as 1-2 h. Ironically, this study revealed a lack of association between the amount of time it took IR to prepare for intervention and the priority treatment sequence of angioembolization for patients with hemodynamically unstable pelvic fractures. Yet, all participants reported utilization of alternative treatments while IR prepared for intervention. Not surprisingly, circumferential compression device was the most common treatment utilized while waiting; which is non-invasive and easily applied [2]. Pelvic packing was also a common treatment modality utilized while IR prepared; a sequence described by Burlew et al. [9] Almost half the participants indicated REBOA was utilized while IR prepared for intervention, suggesting more widespread use than previously reported [18]. The variety of treatment modalities used while waiting is no surprise, given that no guideline provides direction in this situation [2-6]. Therefore, more data is needed to determine the optimal priority treatment when IR is not prepared for intervention.

\section{Limitations}

The response rate of $25 \%$ was a limitation as the participants responses may not be representative of all Level I trauma centers. The online-only survey format may have negatively impacted the response rate as some trauma medical directors noted a preference towards paper surveys. Some Level I trauma centers had outdated contact information for the trauma medical director which resulted in less email invitations being sent to the participant. Responses may have been subject to self-report and recall biases. Survey anonymity and instructions to have protocols on-hand were precautions to reduce these biases. In addition, mortality data was not collected; therefore we cannot conclude what practices were associated with better outcomes.

\section{Conclusions}

The optimal priority treatment sequence for pelvic fractures has not been definitively determined. The reported IR arrival time and time to prepare for intervention did not significantly predict the priority treatment sequence of angioembolization; suggesting the priority treatment sequence was not altered based on these timing metrics. The use of angioembolization first may only be viable to prevent mortality at centers with 24-h on-site IR availability or faster preparation times. Level I trauma centers should review the literature and guidelines on time to angioembolization, their own arrival times for interventional radiology when working off-site, and their intervention preparation times for angioembolization to ensure utilization of the treatment options in an optimal sequence for patient survival. 


\section{Appendix}

Level I Trauma Centers Invited to Participate in the Survey

Albany Medical Center

Banner University Medical Center - Tucson

Banner University Medical Center Phoenix

Barnes-Jewish Hospital

Baylor University Medical Center at Dallas

Baystate Medical Center

Beaumont Hospital - Royal Oak Campus

Bellevue Hospital Center

Ben Taub Hospital - Harris Health System

Beth Israel Deaconess Medical Center

Boston Medical Center

Brigham and Women's Hospital

Bronson Methodist Hospital

Brooke Army Medical Center

Carilion Roanoke Memorial Hospital

Carolinas Medical Center

Cedars-Sinai Medical Center

Charleston Area Medical Center

Christiana Care Health System

Cleveland Clinic Akron General

Community Regional Medical Center

Cooper University Health Care

Dartmouth-Hitchcock Medical Center

Dell Seton Medical Center at the University of Texas

Denver Health Medical Center

Detroit Receiving Hospital

Dignity Health Chandler Regional Medical Center

Dignity Health St. Joseph's Hospital and Medical Center

Duke University Hospital

East Texas Medical Center Tyler

Erie County Medical Center

Eskenazi Health

Froedtert Hospital

George Washington University Hospital

Grady Memorial Hospital

Grant Medical Center

Greenville Memorial Hospital

Harbor UCLA Medical Center

Hartford Hospital

Hennepin County Medical Center

Henry Ford Hospital

Highland Hospital/A member of Alameda Health System

HonorHealth John C. Lincoln Medical Center

HonorHealth Scottsdale Osborn Medical Center

Howard University Hospital

Hurley Medical Center

Indiana University Health Methodist Hospital

Inova Fairfax Hospital

Intermountain Medical Center

lowa Methodist Medical Center

Jackson Memorial Hospital

Jacobi Medical Center

Jamaica Hospital Medical Center

JPS Health Network

Kendall Regional Medical Center

LAC + USC Medical Center

Legacy Emanuel Medical Center

Lincoln Medical and Mental Health Center

Loyola University Medical Center

Maine Medical Center

Maricopa Integrated Health System - Maricopa Medical Center

Massachusetts General Hospital

\section{Appendix (Continued)}

Mayo Clinic Rochester Trauma Centers

Medical Center Navient Health

Medical University of South Carolina

MedStar Washington Hospital Center

Memorial Hermann Hospital System - Houston

Memorial Regional Hospital

Mercy Health - St. Elizabeth Youngstown Hospital

Mercy Health - St. Vincent Medical Center

Methodist Dallas Medical Center

MetroHealth Medical Center

Miami Valley Hospital

Morristown Medical Center

Nassau University Medical Center

Nebraska Medicine - Nebraska Medical Center

New Jersey Trauma Center at the University Hospital

New York Presbyterian Hospital - Weill Cornell Medical Center

New York-Presbyterian - Queens

North Memorial Health Hospital

Northwell Health North Shore University Hospital

Northwell Health Staten Island University Hospital

NYC Health and Hospitals - Elmhurst

NYC Health and Hospitals - Kings County

NYU Langone Hospital - Brooklyn

NYU Winthrop Hospital

Oregon Health \& Science University

OU Medical Center

Palmetto Health Richland

Parkland Health \& Hospital System

Penrose Hospital

ProMedica Toledo Hospital

Regions Hospital

Rhode Island Hospital

Richmond University Medical Center

Robert Wood Johnson University Hospital

Ronald Reagan UCLA Medical Center

Santa Barbara Cottage Hospital

Santa Clara Valley Medical Center

Scott \& White Memorial Hospital - Temple

Scripps Mercy Hospital

Sparrow Hospital

Spectrum Health - Butterworth Hospital

SSM Health Saint Louis University Hospital

St. Anthony Hospital

St. Joseph Mercy Hospital - Ann Arbor

St. Vincent Indianapolis Hospital

Stanford Health Care

Stony Brook Medicine

Summa Akron City Hospital

Swedish Medical Center

Tampa General Hospital

The Medical Center of Plano

The Ohio State University Wexner Medical Center

The Queen's Medical Center

The University of Kansas Hospital

The University of Toledo Medical Center

Tufts Medical Center

UC Irvine Health

UC San Diego Medical Center

UMASS Memorial Medical Center

University Health System - San Antonio

University Health-Shreveport

University Hospitals Cleveland Medical Center

University Medical Center - Lubbock

University Medical Center New Orleans 


\section{Appendix (Continued)}

University Medical Center of El Paso

University Medical Center of Southern Nevada

University of Alabama at Birmingham Hospital

University of Arkansas for Medical Sciences

University of California, Davis Medical Center

University of Cincinnati Medical Center

University of lowa Hospitals \& Clinics

University of Kentucky Albert B. Chandler Hospital

University of Louisville Hospital

University of Michigan Health System

University of Missouri Health System

University of New Mexico Hospital

University of North Carolina Hospital

University of Rochester Medical Center/Strong Memorial Hospital

University of Tennessee Medical Center

University of Texas Medical Branch

University of Utah Health Care

University of Vermont Medical Center

University of Virginia Health System

University of Wisconsin Hospital and Clinics Authority

Upstate University Hospital

Vanderbilt University Medical Center

Via Christi Hospitals - Wichita

Vidant Medical Center

Virginia Commonwealth University Medical Center

Wake Forest Baptist Medical Center

WakeMed Health \& Hospitals

Wesley Medical Center

West Virginia University Hospitals-J.W. Ruby Memorial Hospital

Westchester Medical Center

Yale-New Haven Hospital

Zuckerberg San Francisco General Hospital and Trauma Center

\section{Abbreviations}

ACS: American College of Surgeons; ATLS: Advanced Trauma Life Support; EAST: Eastern Association for the Surgery of Trauma; IR: Interventional Radiology; REBOA: Resuscitative Endovascular Balloon Occlusion of the Aorta; TQIP: Trauma Quality Improvement Program; WSES: World Society of Emergency Surgeons; WTA: Western Trauma Association

\section{Acknowledgements}

We would like to thank all the participating Trauma Medical Directors who shared their time, experience, and protocol information for this survey.

\section{Authors' contributions}

SJ contributed to conception and study design, acquisition of data, analyzed and interpreted the data, drafted and revised the manuscript, and agreed to be accountable for all aspects of the work. AO contributed to conception and study design, critically revised manuscript, provided final approval of the manuscript submitted, and agreed to be accountable for all aspects of the work. $\mathrm{BB}, \mathrm{KB}, \mathrm{CR}, \mathrm{GB}, \mathrm{NP}, \mathrm{MK}, \mathrm{MC}$, and $\mathrm{DBO}$ contributed to conception and study design, interpreted the data, critically revised manuscript, provided final approval of the manuscript submitted. All authors read and approved the final manuscript.

\section{Funding}

Not applicable.

\section{Availability of data and materials}

Data for this study is stored on Sharefile, an electronic HIPAA and HITECHcompliant platform that ensures all transmissions are fully encrypted, end-toend. The datasets used for analysis for the current study are available from the corresponding author on reasonable request.

\section{Ethics approval and consent to participate}

This study was approved by Western Institutional Review Board, IRB Study No: 1183667. Western Institutional Review Board Multiple Project Assurance Number: IRB00000533.

The study was approved with a partial waiver of consent, waiving the requirement for a conform containing a signature of the participant.

\section{Consent for publication}

Not applicable.

\section{Competing interests}

The authors declare that they have no competing interests.

\section{Author details}

${ }^{1}$ Trauma Research, LLC, 383 Corona St. \#319, Denver, CO 80218, USA.

${ }^{2}$ Research Medical Center, 2316 East Meyer Blvd, Kansas City, MO 64132, USA

${ }^{3}$ University of Connecticut, Hartford Hospital, Hartford, CT 06106, USA.

${ }^{4}$ Swedish Medical Center, 501 E Hampden Ave, Englewood, CO 80113, USA

${ }^{5}$ Wesley Medical Center, 550 N. Hillside St, Wichita, KS 67214, USA. ${ }^{6}$ St.

Anthony's Hospital, 11600 West 2nd Place, Lakewood, CO 80228, USA.

${ }^{7}$ Penrose Hospital, 2222 North Nevada Ave, Colorado Springs, CO 80907,

USA. ${ }^{8}$ Medical City Plano, 3901 West 15th Street, Plano, TX 75075, USA.

Received: 8 February 2019 Accepted: 27 May 2019

Published online: 20 June 2019

\section{References}

1. Stahel PF, Burlew CC, Moore EE. Current trends in the management of hemodynamically unstable pelvic ring injuries. Curr Opin Crit Care. 2017;23: 511-9.

2. Coccolini F, Stahel PF, Montori G, Biffl W, Horer TM, Catena F, Kluger Y, Moore EE, Peitzman AB, Ivatury $R$, Coimbra R, Fraga GP, Pereira B, Rizoli $S$, Kirkpatrick A, Leppaniemi A, Manfredi R, Magnone S, Chiara O, Solaini L, Ceresoli M, Allievi N, Arvieux C, Velmahos G, Balogh Z, Naidoo N, Weber D, Abu-Zidan F, Sartelli M, Ansaloni L. Pelvic trauma: WSES classification and guidelines. World J Emerg Surg. 2017:12:1-18.

3. Biffl WL, Cothren CC, Moore EE, Kozar R, Cocanour C, Davis JW, Mclntyre RC, West MA, Moore FA. Western trauma association critical decisions in trauma: screening for and treatment of blunt cerebrovascular injuries. J Trauma. 2009:67:1150-3.

4. American College of Surgeons. Best practices in the Management of Orthopaedic Trauma; 2015. p. 1-40. Available from: https://www.facs.org/ / media/files/quality programs/trauma/tqip/traumatic brain injury guidelines. ashx. [Accessed 7 Mar 2018]

5. Cullinane DC, Schiller HJ, Zielinski MD, Bilaniuk JW, Collier BR, Como J, Holevar M, Sabater EA, Sems SA, Vassy WM, Wynne JL. Eastern Association for the Surgery of trauma practice management guidelines for hemorrhage in pelvic fracture-update and systematic review. J Trauma. 2011;71:1850-68.

6. The American College of Surgeons. Advanced trauma life support (ATLS $\left.{ }^{\oplus}\right)$ : the ninth edition. Chicago: American College of Surgeons; 2013.

7. Tanizaki S, Maeda S, Matano H, Sera M, Nagai H, Ishida H. Time to pelvic embolization for hemodynamically unstable pelvic fractures may affect the survival for delays up to 60 min. Injury. 2014;45:738-41 Elsevier Ltd.

8. The American College of Surgeons. In: Rotondo MF, Cribara C, Smith RS, editors. Resources for optimal care of the injured patient. 6th ed. Chicago: American College of Surgeons; 2014.

9. Burlew CC, Moore EE, Smith WR, Johnson JL, Biffl WL, Barnett CC, Stahel PF. Preperitoneal pelvic packing/external fixation with secondary angioembolization: optimal care for life-threatening hemorrhage from unstable pelvic fractures. J Am Coll Surg. 2011;212:628-35 Elsevier Inc.

10. Blondeau B, Orlando A, Jarvis S, Banton K, Berg GM, Patel N, Meinig R, Tanner A, Carrick M, Bar-Or D. Variability in pelvic packing practices for hemodynamically unstable pelvic fractures at US level 1 trauma centers. Patient Saf Surg. 2019;13:1-10.

11. Suzuki T, Smith WR, Moore EE. Pelvic packing or angiography: competitive or complementary? Injury. 2009;40:343-53.

12. Smith WR, Moore EE, Osborn P, Agudelo JF, Morgan SJ, Parekh AA, Cothren C. Retroperitoneal packing as a resuscitation technique for hemodynamically unstable patients with pelvic fractures: report of two representative cases and a description of technique. J Trauma. 2005;59:1510-4. 
13. Burlew CC, Moore EE, Stahel PF, Geddes AE, Wagenaar AE, Pieracci FM, Fox CJ, Campion EM, Johnson JL, Mauffrey C. Preperitoneal pelvic packing reduces mortality in patients with life-threatening hemorrhage due to unstable pelvic fractures. J Trauma Acute Care Surg. 2017:82:233-42.

14. Tai DKC, Li W-H, Lee K-Y, Cheng M, Lee K-B, Tang L-F, Lai AK-H, Ho H-F, Cheung M-T. Retroperitoneal pelvic packing in the Management of Hemodynamically Unstable Pelvic Fractures: a level I trauma center experience. J Trauma. 2011;71:E79-86.

15. Osborn PM, Smith WR, Moore EE, Cothren CC, Morgan SJ, Williams AE, Stahel PF. Direct retroperitoneal pelvic packing versus pelvic angiography: a comparison of two management protocols for haemodynamically unstable pelvic fractures. Injury. 2009;40:54-60.

16. Li Q, Dong J, Yang Y, Wang G, Wang Y, Liu P, Robinson Y, Zhou D.

Retroperitoneal packing or angioembolization for haemorrhage control of pelvic fractures - quasi-randomized clinical trial of 56 haemodynamically unstable patients with injury severity score $\geq 33$. Injury. 2016;47:395-401 Elsevier Ltd.

17. Cothren CC, Osborn PM, Moore EE, Morgan SJ, Johnson JL, Smith WR. Preperitonal pelvic packing for hemodynamically unstable pelvic fractures: a paradigm shift. J Trauma. 2007;62:834-42.

18. Stannard A, Eliason JL, Rasmussen TE. Resuscitative endovascular balloon occlusion of the aorta (REBOA) as an adjunct for hemorrhagic shock. Trauma. 2011;71:1869-72

\section{Publisher's Note}

Springer Nature remains neutral with regard to jurisdictional claims in published maps and institutional affiliations.

Ready to submit your research? Choose BMC and benefit from:

- fast, convenient online submission

- thorough peer review by experienced researchers in your field

- rapid publication on acceptance

- support for research data, including large and complex data types

- gold Open Access which fosters wider collaboration and increased citations

- maximum visibility for your research: over $100 \mathrm{M}$ website views per year

At BMC, research is always in progress.

Learn more biomedcentral.com/submissions 